\title{
Ciclo de mejora en Ingeniería de la Prevención de Riesgos Laborales
}

\section{Improvement cycle in Prevention Engineering of Occupational Risk}

ISABEL MONTEALEgRE MELÉNDEZ

https://orcid.org/0000-0001-8598-0578

Universidad de Sevilla

Departamento de Ciencia e Ingeniería

de los Materiales y del Trasporte

imontealegre@us.es

DOI: http://dx.doi.org/10.12795/9788447231003.048

Pp.: 1004-1025 


\section{Introducción y contexto de la asignatura}

En este trabajo se presenta el Ciclo de Mejora en el Aula (CIMA) realizado para la asignatura de Ingeniería de la Prevención de Riesgos Laborales (IPRL), del Grado en Ingeniería Mecánica en el 4 o curso, siendo una asignatura obligatoria y que también se da de forma transversal en el Doble Grado en Ingeniería Eléctrica e Ingeniería Mecánica y el Doble Grado en Ingeniería en Diseño Industrial y Desarrollo del Producto e Ingeniería Mecánica. Esta asignatura es impartida por el departamento de Ciencia e Ingeniería de los Materiales. Debido a las limitaciones causadas por la pandemia, la parte teórica de la asignatura ha sido impartida de forma telemática desde el comienzo del curso 2020/2021; sin embargo las prácticas de laboratorio si se han realizado presencialmente.

En el ciclo se ha trabajado con un alto número de estudiantes (111 alumnos matriculados); la cifra ha sido más elevada en comparación con años anteriores que rozaban los 80 estudiantes en un único grupo. Este hecho ha resultado un gran reto para la aplicación del CIMA. La profesora que lo ha llevado a cabo, lleva al frente de esta materia desde el comienzo de la aparición del Grado de Mecánica. Es importante destacar el bajo número de alumnos repetidores en esta materia ( 2 alumnos). Con ello se pone de manifiesto el contexto de la asignatura donde existe una gran ratio de alumnos-docente.

Hay que destacar que el contenido de la asignatura (temario) consta de 5 Bloques temáticos. El CIMA realizado fue en el Bloque 2 correspondiente a la parte de la temática de Seguridad en el Trabajo; comprendiendo 5 temas de este segundo bloque.

Ciclos de Mejora en el Aula (2020). Experiencias de Innovación Docente de la US Esta obra se distribuye con la licencia Creative Commons 


\section{Diseño previo del CIMA}

Previo a la aplicación del CIMA, se realizó un diseño en base a las directrices más convenientes para el desarrollo del mismo; para ello se valoraron diferentes factores que pudiesen influir positivamente en el CIMA. Partir de un gran número de alumnos con una asignatura que hasta el curso 2020/2021 se había ido impartiendo mediante una metodología tradicional, con dificultad de trabajar en grupos reducidos y gran contenido teórico, suponía un gran reto a superar. Mediante la aplicación del CIMA se esperaba un cambio substancial; era deseable que los alumnos conectaran con la asignatura y sus contenidos, y a la vez la profesora fuera una buena docente tratando de llegar a todos y cada uno de los alumnos matriculados en la asignatura.

\section{Modelo Metodológico y Mapa de Contenidos}

Como se aprecia en la Figura 1, se partían de clase tipo basadas en teoría en un $75 \%$ y un $25 \%$ en la resolución de casos prácticos o ejercicios. Esta situación es algo que con la ejecución del ciclo de mejora se pretende cambiar. Es importante plantearse el contexto de aprendizaje adecuado para los estudiantes (Bain, 2007), y para ello hay que trabajar mejorando el modelo metodológico y planificando minuciosamente un mapa de contenidos, en los que quede claro que tipos de contenidos son fundamentales y cuáles no.

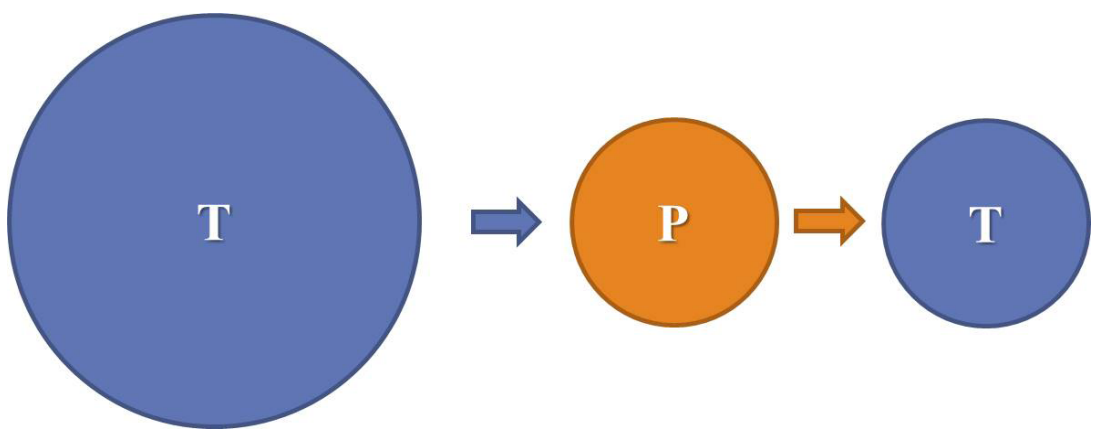

Figura 1. Modelo metodológico habitual

Ciclos de Mejora en el Aula (2020). Experiencias de Innovación Docente de la US Esta obra se distribuye con la licencia Creative Commons 


\section{Mapa de Contenidos}

Los contenidos que se propusieron dar durante la ejecución del CIMA quedan reflejados en la Figura 2. El orden en el que aparecían en el programa de la asignatura de IPRL fue alterado con el fin de alcanzar una mayor conexión entre los mismos. Al encontrarnos en el ámbito de la Seguridad en el trabajo, el accidente laboral es el punto de partida, siendo las técnicas de seguridad el eje central de los contenidos. Los contenidos actitudinales fueron los pilares en los que se basó el CIMA. Se le dio mucha importancia a que los alumnos asimilasen no sólo contenidos conceptuales, sino procedimentales; por ello, siguiendo directrices ilustradas en la bibliografía (Finkel, 2000) todas las referencias en el texto siguiendo normas APA, se plantearon actividades de diversa tipología. Con el objetivo de fomentar la participación de los alumnos en clase, los talleres que se fijaron fueron todos grupales; de este modo los estudiantes intervendrían en grupos pequeños y posteriormente, se buscaría la forma de realizar una participación dentro del grupo principal. La profesora tendría en determinadas ocasiones un papel secundario, permitiendo que los alumnos expusieran sus opiniones en torno al tema planteado en cada actividad. Esto se verá en el siguiente apartado.

Ciclos de Mejora en el Aula (2020). Experiencias de Innovación Docente de la US Esta obra se distribuye con la licencia Creative Commons 


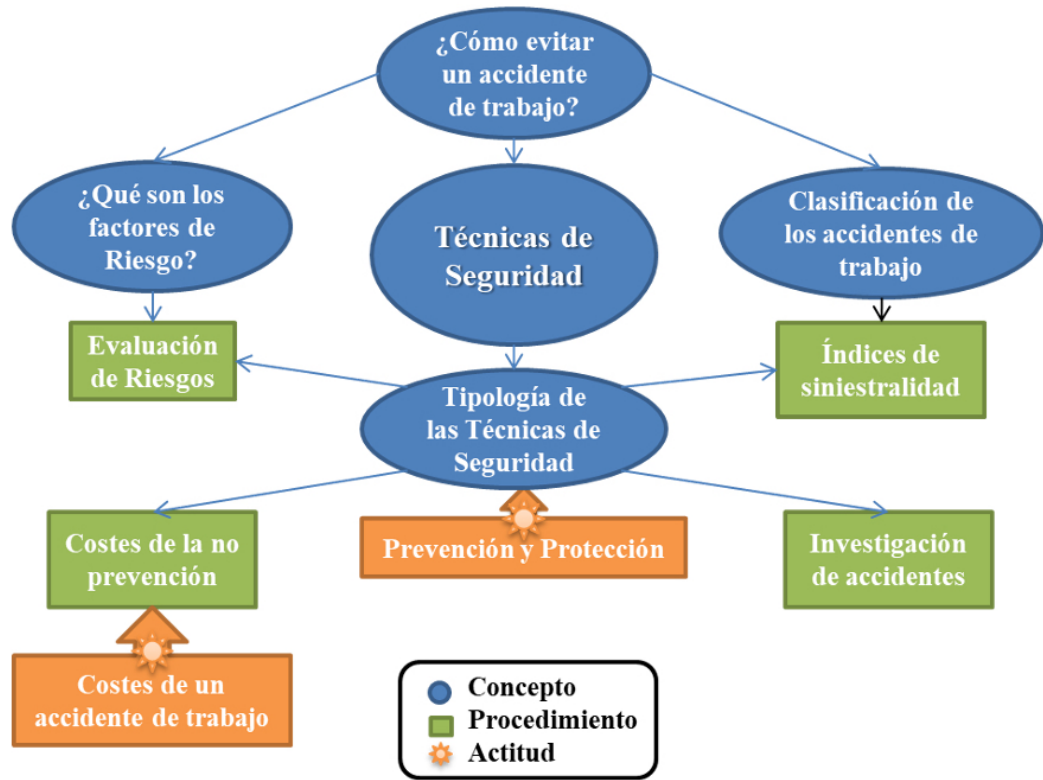

Figura 2. Mapa de contenidos

\section{Modelo Metodológico}

Fue todo un reto tratar de incorporar actividades diferentes, que modificaran significativamente la metodología tradicional que se venía realizando hasta la aplicación del CIMA; el modelo a seguir se basó fundamentalmente en potenciar la participación de los estudiantes. Se realizarían talleres, donde los alumnos formando grupos reducidos, trabajarían siguiendo las directrices pertinentes. Con estos talleres los propios alumnos podrían llegar a descubrir conocimientos ya adquiridos en temas anteriores y plantearían dudas que serían resueltas por ellos mismos bajo la guía de la profesora.

En la Figura 3, se pueden apreciar la secuencia de pasos que se plantearon antes del ciclo de mejora. Las actividades estaban concebidas con una duración de 1 hora, salvo una actividad; esto se explica en detalle en el siguiente apartado.

Ciclos de Mejora en el Aula (2020). Experiencias de Innovación Docente de la US Esta obra se distribuye con la licencia Creative Commons 


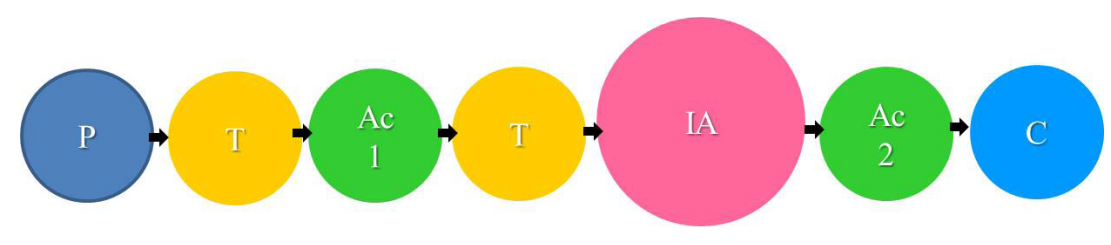

Figura 3. Modelo metodológico diseñado para el CIMA. P: PresentaciónPregunta clave; Ac: Actividad de Contraste; T: Teoría; IA: Ideas de los alumnos; $\mathrm{C}$ : conclusiones (T-C)

\section{Secuencia de actividades}

Antes de comenzar el ciclo de mejora diseñado, se pasó un cuestionario a los alumnos; seguidamente se empezó el CIMA con la primera actividad. La secuencia de actividades se detalla minuciosamente a continuación.

Tabla 1. Secuencia de actividades del Ciclo de Mejora en el Aula

\begin{tabular}{|l|c|c|}
\hline \multicolumn{3}{|c|}{ SESIÓN 1 } \\
\hline \multicolumn{3}{|c|}{ ACCIDENTES DE TRABAJO } \\
\hline Act. 1 & \multicolumn{3}{|c|}{ PRESENTACIÓN Y EXPLICACIÓN } & 10 min & P \\
\hline $\begin{array}{l}\text { Descripción de la actividad } \\
\text { Se presentará el comienzo del CIMA; se realizará una explicación por parte } \\
\text { de la profesora del desarrollo de la actividad y de la forma de trabajo. Se les } \\
\text { indicará a los alumnos cómo se plantea la sesión 1. } \\
\text { Primero se partirá de unas preguntas, realizadas a todos los alumnos en } \\
\text { la sesión principal, y posteriormente se trabajará la actividad en sesiones } \\
\text { grupales. Se explicará que se van a formar grupos de } 6 \text { alumnos como } \\
\text { máximo. Los estudiantes podrán elegir a los compañeros del grupo y } \\
\text { designarán un portavoz, para una puesta en común que se realizará al final } \\
\text { de la sesión 1. La profesora comentará la importancia que tiene el hecho } \\
\text { de que ellos participen y la realicen en el tiempo propuesto para ello. Les } \\
\text { comunicará que ella irá resolviendo dudas y trabajando con cada grupo de } \\
\text { forma individual. La gestión de la actividad realizada de forma virtual con } \\
\text { 111 alumnos puede dilatar la duración de la actividad, por lo que es muy } \\
\text { importante la puntualidad y el compromiso de los alumnos. }\end{array}$ \\
\hline
\end{tabular}

Ciclos de Mejora en el Aula (2020). Experiencias de Innovación Docente de la US Esta obra se distribuye con la licencia Creative Commons Reconocimiento-NoComercial-SinObraDerivada Internacional (CC BY-NC-ND 4.0.) 


$$
\begin{gathered}
\text { Act. } 2 \\
\begin{array}{c}
\text { PREGUNTA CLAVE: ¿Cómo se evitan los accidentes } \\
\text { de trabajo? }
\end{array}
\end{gathered}
$$

\section{Descripción de la actividad}

Antes de formar los grupos mediante la herramienta pertinente en Blackboard Collaborate Ultra, la profesora expondrá el problema en la sesión central que posteriormente los alumnos discutirán de forma grupal: ¿cómo se evitan los accidentes de trabajo?, ¿todos los accidentes de trabajo son iguales?, ¿hay diferencias entre ellos?

Tras formular las preguntas, la profesora indicará a los alumnos que las instrucciones de la actividad están recogidas en un guion subido previamente en la plataforma. En este momento deja de estar todo el grupo de alumnos juntos en la sala virtual principal y se forman los grupos que permite la herramienta del Blackboard Collaborate Ultra (20 grupos de trabajo)

\begin{tabular}{|c|c|c|c|}
\hline Act. 3 & $\begin{array}{c}\text { PREGUNTA CLAVE: ¿Cómo se evitan los accidentes } \\
\text { de trabajo? ¿Tipos de accidentes de trabajo? }\end{array}$ & $30 \mathrm{~min}$ & $\mathrm{~A}$ \\
\hline
\end{tabular}

Una vez comenzado el bloque temático dedicado a la Seguridad Laboral, el punto central del mismo se focaliza en el estudio de los accidentes de trabajo; por ello la profesora planteará a cada grupo que respondan e indiquen ejemplos entorno a las distintas categorías de accidente de trabajo. Los alumnos deben responder de forma grupal buscando ejemplos que ellos mismo inventen o que encuentre de diversas fuentes o medios informativos. La profesora previamente ha realizado un guion, y lo ha puesto a disposición de los alumnos en la plataforma. Los alumnos disponen de tiempo para poder realizar la actividad. La profesora entrará de forma virtual en todos los grupos como mera oyente.

Cada grupo de trabajo debe explicar y dar ejemplos "reales" o "ficticios" de los tipos de AT que se muestran en la siguiente lista:

1. Accidente "in itinere"

2. Accidente en misión y de cargos electivos de carácter sindical

3. Accidente en tareas no habituales por decisión ajena

4. Accidente en actos de salvamento

5. Enfermedad o defectos anteriores*

6. a) Enfermedad intercurrentes

6. a) Enfermedades comunes que contraiga el trabajador/a con motivo de la realización de su trabajo

7. Debidos a imprudencias profesionales

\begin{tabular}{|l|l|l|l|}
\hline Act. 3 & PUESTA EN COMÚN & $10 \mathrm{~min}$ & $\operatorname{Pr}$ \\
\hline
\end{tabular}

Antes de terminar la sesión los alumnos que hayan tenido una duda y no haya sido resuelta la podrán poner en común antes de que finalice la sesión.

Recurso: Se empleará cuestionario subido a la plataforma y la herramienta Blackboard Collaborate Ultra.

Ciclos de Mejora en el Aula (2020). Experiencias de Innovación Docente de la US Esta obra se distribuye con la licencia Creative Commons Reconocimiento-NoComercial-SinObraDerivada Internacional (CC BY-NC-ND 4.0.) 
Recurso: Diapositivas de ppt y la herramienta Blackboard Collaborate Ultra. 


\begin{tabular}{|c|c|c|c|}
\hline \multicolumn{4}{|c|}{ SESIÓN 3} \\
\hline \multicolumn{4}{|c|}{ PRGUNTA CLAVE: INDICES DE SINIESTRALIDAD ¿CÓMO SE CALCULAN? } \\
\hline Act. 6 & PREPARACIÓN Y PLANTEAMIENTO & $10 \mathrm{~min}$ & $\operatorname{Pr}$ \\
\hline \multicolumn{4}{|c|}{$\begin{array}{l}\text { Descripción de la actividad } \\
\text { Explicación de cómo se va a desarrollar la sesión. Se explicará que su } \\
\text { desarrollo va a ser grupal y se formarán } 20 \text { grupos con un máximo } 6 \text { alumnos. } \\
\text { Los alumnos en la sesión anterior descubrieron la existencia de las técnicas } \\
\text { estadísticas para la "previsión de accidentes de trabajo". La profesora } \\
\text { comentará la importancia de saber realizar el cálculo pertinente de los } \\
\text { indices estadísticos más representativos en seguridad laboral. Mediante } \\
\text { una dinámica de grupo, anteriormente ya comentada, los alumnos con la } \\
\text { documentación teórica vista en la sesión anterior, realizará la actividad. La } \\
\text { profesora estará a disposición de los alumnos y ayudando de forma virtual } \\
\text { a aquellos grupos que tengan problemas a la hora de realizar los cálculos. }\end{array}$} \\
\hline Act. 7 & EJERCICIO-TRABAJO EN GRUPOS & $45 \mathrm{~min}$ & AC \\
\hline \multicolumn{4}{|c|}{$\begin{array}{l}\text { Descripción de la actividad } \\
\text { Se trabajará de forma grupal en las sesiones de trabajo de grupos en } \\
\text { Blackboard Collaborate Ultra. La profesora estará visitando de forma virtual } \\
\text { a los distintos grupos, tratando de corregir posibles errores de cálculo y } \\
\text { resolviendo dudas. }\end{array}$} \\
\hline Act. 8 & PUESTA EN COMÚN & $5 \mathrm{~min}$ & A \\
\hline \multicolumn{4}{|c|}{$\begin{array}{l}\text { Descripción de la actividad } \\
\text { Exposición por parte de los portavoces de los distintos grupos acerca de los } \\
\text { resultados obtenidos y su significado. }\end{array}$} \\
\hline Recurso & Is ppt y hoja de cálculo de exce & & \\
\hline
\end{tabular}

\begin{tabular}{|c|c|c|c|}
\hline \multicolumn{4}{|c|}{ SESIÓN 4} \\
\hline \multicolumn{4}{|c|}{$\begin{array}{c}\text { FUNDAMENTOS TEÓRICOS SOBRE INVESTIGACIÓN DE ACIDENTES DE TRABAJO } \\
\text { Y SUS COSTES }\end{array}$} \\
\hline Act. 9 & & $5 \mathrm{~min}$ & $\operatorname{Pr}$ \\
\hline \multicolumn{4}{|c|}{$\begin{array}{l}\text { Descripción de la actividad } \\
\text { Se realizará un repaso del contenido abordado en la clase anterior y se } \\
\text { solucionarán las dudas que puedan tener los alumnos. } \\
\text { Luego se procederá a la explicación de cómo se va a desarrollar la sesión } \\
\text { principalmente con carácter teórico. Se explicará la metodología aplicada } \\
\text { a la investigación de accidentes de trabajo y al cálculo de los costes de los } \\
\text { mismos. La profesora tratará de abrir un breve debate entre los alumnos. }\end{array}$} \\
\hline Act.10 & DEBATE & $15 \mathrm{~min}$ & IA \\
\hline
\end{tabular}

Ciclos de Mejora en el Aula (2020). Experiencias de Innovación Docente de la US Esta obra se distribuye con la licencia Creative Commons 
Descripción de la actividad

En la sala principal, la profesora expone si es rentable un accidente de trabajo. Se espera que los alumnos participen. La profesora actuará meramente como oyente. Transcurrido el tiempo destinado a esta actividad se procederá a la descripción teórica de las fórmulas para el cálculo de los costes de los accidentes de trabajo.

\begin{tabular}{|c|c|c|c|}
\hline Act.11 & $\begin{array}{c}\text { DESCRIPCIÓN TEÓRICA Y } \\
\text { CONCLUSIONES }\end{array}$ & 30 min & T-C \\
\hline \multicolumn{4}{|c|}{ Explicación de las teorías de cálculo de costes de accidentes de trabajo. } \\
\hline Act.12 & PROBLEMA PROPUESTO & 15 min & pR \\
\hline
\end{tabular}

Se propondrá un ejercicio para que los alumnos lo realicen de forma individual, basado en la teoría vista en esta sesión. Las dudas surgidas durante la realización del mismo serán expuestas por los alumnos en tutorías o al comienzo de la siguiente sesión.

Recurso: diapositivas ppt y hoja de cálculo de excel

\begin{tabular}{|c|c|c|c|}
\hline \multicolumn{4}{|c|}{ SESIÓN 5 } \\
\hline \multicolumn{4}{|c|}{ INVESTIGACIÓN DE ACCIDENTE DE TRABAJO } \\
\hline ACt.13 & $\begin{array}{c}\text { PRESENTACIÓN Y DESCRIPCIÓN DE LA } \\
\text { ACTIVIDAD }\end{array}$ & 10 min & Pr \\
\hline
\end{tabular}

Descripción de la actividad

En la sala principal, la profesora expone brevemente la importancia de la técnica de seguridad "investigación de accidentes" y explica la metodología más común a la hora de investigar uno: el método del árbol de causas. Esta información es necesaria para la realización de la IA (actividad de expresión de las Ideas de los Alumnos). Se propone la creación de los grupos de trabajo en Blackboard Collaborate Ultra con un máximo de 6 estudiantes que ellos mismos decidirán, y establecerán un portavoz del grupo. Se expone la dinámica de la sesión y se les indica a los alumnos que accedan a la plataforma y elijan un accidente de trabajo por cada grupo. Esta documentación previamente ha sido colgada en la carpeta del tema.

La profesora procede a formar grupos de trabajo virtuales en lo que se trabajará la IA. No importa si hay más de un grupo trabajando sobre el mismo accidente.

\begin{tabular}{|c|c|c|c|}
\hline Act.14 & TRABAJO EN GRUPOS & $50 \mathrm{~min}$ & IA \\
\hline
\end{tabular}

Ciclos de Mejora en el Aula (2020). Experiencias de Innovación Docente de la US Esta obra se distribuye con la licencia Creative Commons 
Descripción de la actividad

Una vez designados los grupos, trabajarán en la tarea durante el tiempo asignado, consensuando una respuesta común. Toda la IA girará en la investigación de un accidente de trabajo, que previamente han elegido los componentes del grupo. La metodología a seguir ha sido descrita previamente y además los alumnos tiene toda la documentación a su disposición en la carpeta de contenido de la asignatura. La profesora no interviene en nada; se queda en la sala principal de Blackboard Collaborate Ultra, por si surge algún problema durante la realización de la actividad.

La actividad finalizará con una exposición breve de las conclusiones de cada grupo. El hecho de que varios grupos trabajen por azar en el mismo accidente, es considerado enriquecedor, ya que los propios alumnos reflexionan sobre los distintos resultados obtenidos y el hecho de no valorar aspectos que otros grupos hayan valorado.

\begin{tabular}{l|l|l|l} 
Act.15 & EXPOSICIÓN, DEBATE Y CONCLUSIONES & $60 \mathrm{~min}$ & A
\end{tabular}

Descripción de la actividad

Una vez los alumnos hayan realizado sus investigaciones, y hayan sido capaces de llegar por si mismos a unas conclusiones aplicando la metodología indicada, se exponen sus resultados.

En este caso, cada componente del grupo comenta parte de los resultados de la investigación; la profesora trata de indicar las mejoras que fueran necesarias para unificar todos los resultados obtenidos por varios grupos sobre un mismo accidente investigado.

El alto número de alumnos puede limitar la actividad, pudiendo afectar a la falta de participación por límite de tiempo.

Recurso: Diapositivas de ppt y la herramienta Blackboard Collaborate Ultra.

\begin{tabular}{|c|c|c|}
\hline \multicolumn{3}{|c|}{ SESIÓN 6 } \\
\hline \multicolumn{4}{|c|}{ EVALUACIÓN DE RIESGOS } \\
\hline Act.16 & 10 min & Pr \\
\hline $\begin{array}{l}\text { Descripción de la actividad } \\
\text { Explicación de la metodología empleada para la realización de una evaluación } \\
\text { de riesgos. La profesora previamente subirá a la carpeta de contenidos de la } \\
\text { asignatura descriptores en los que se basa la realización de la actividad. Se } \\
\text { detallará el procedimiento de la misma. Se formarán grupos habituales de } \\
\text { trabajo de un máximo de } 6 \text { estudiantes, con un portavoz como representante; } \\
\text { los propios alumnos pueden formarlos. Para todos los grupos se indicará un } \\
\text { caso de estudio y la profesora participará en la resolución del problema a } \\
\text { evaluar. }\end{array}$ \\
\hline Recursos: Diapositivas pp & 40 min & A \\
\hline \multicolumn{3}{|c|}{ TRABAJO EN GRUPOS }
\end{tabular}

Ciclos de Mejora en el Aula (2020). Experiencias de Innovación Docente de la US Esta obra se distribuye con la licencia Creative Commons Reconocimiento-NoComercial-SinObraDerivada Internacional (CC BY-NC-ND 4.0.) 
Descripción de la actividad

Los estudiantes realizarán bajo la supervisión de la profesora una evaluación de riesgos muy elemental. Para aquel grupo que lo desee, la profesora recomendará casos más complejos de estudio. Trascurrido el tiempo, todos los alumnos volverán a la sala principal.

\begin{tabular}{l|l|l|l}
\hline Act.18 & PUESTA EN COMÚN-CONCLUSIONES & $10 \mathrm{~min}$ & A-C \\
\hline
\end{tabular}

Descripción de la actividad

Se describirá brevemente los resultados obtenidos, que en su mayoría deberán coincidir. Se planteará supuestos prácticos a realizar por los alumnos que lo deseen para una mayor profundización sobre el tema, ya que la evaluación de riesgos es una técnica clave en seguridad en el trabajo.

Recurso: diapositivas ppt y recursos digitales sobre accidentes de trabajo

\begin{tabular}{|l|l|l|l|}
\hline \multicolumn{4}{|c|}{ SESIÓN 8} \\
\hline Act. 19 & CONCLUSIONES DEL CIMA & $35 \mathrm{~min}$ & $\mathrm{C}$ \\
\hline
\end{tabular}

Descripción de la actividad

Para concluir con el CIMA se realizará una puesta en común donde los alumnos podrán valorar los aspectos aprendidos durante la aplicación del mismo. Mediante un sondeo en Blackboard Collaborate Ultra, se tratará de valorar si los alumnos han asimilado los conceptos.

Los propios alumnos que lo deseen pueden realizar preguntas al resto de sus compañeros. De este modo, los estudiantes se pueden motivar a participar en esta actividad.

Recurso: Cuestionario Kahoot!

\begin{tabular}{|c|c|c|c|}
\hline Act. 20 & CUESTIONARIO FINAL & $25 \mathrm{~min}$ & $\mathrm{C}$ \\
\hline
\end{tabular}

Descripción de la actividad

Se les comunicará a los estudiantes la realización del mismo sondeo que realizaron antes de la aplicación del CIMA.

Recurso: Cuestionario y Blackboard Collaborate Ultra

\section{Cuestionario inicial/final}

Como se ha indicado al comienzo, antes de la aplicación del ciclo de mejora, a los alumnos se les pasó un cuestionario basado en los contenidos que se iba a ver durante las sesiones. Los alumnos de forma anónima lo rellenaron y lo entregaron; los resultados del mismo fueron analizados y de ellos se extrajeron unas conclusiones que se explicaran en el siguiente apartado. A continuación se presenta el cuestionario:

Ciclos de Mejora en el Aula (2020). Experiencias de Innovación Docente de la US Esta obra se distribuye con la licencia Creative Commons 
Introducción: Somos el equipo de seguridad y riesgos laborales de la empresa "Construcciones Imposibles, S.A." y estamos construyendo un puente ajardinado entre Sevilla y Nueva York en pleno mes de agosto. Lleva preparadas tus respuestas al cuestionario para la próxima reunión, pues tenemos que hacer el informe mensual a la gerencia. Quiero que te expreses con el máximo de libertad y con tus puntos de vista, asi que este sondeo es anónimo y lo debatiremos en la reunión sin saber quién lo ha escrito. Argumenta muy bien todas tus opiniones y pon una clave para que puedas recuperar tu cuestionario al final de la reunión. Gracias. Coordinadora del Equipo de Seguridad.

1. ¿Cómo podemos evitar al máximo posible los tipos de accidente de trabajo que se pueden dar en la construcción del puente? Explica con detalle tus propuestas

2. ¿Qué son para ti las técnicas en seguridad laboral? Debemos ponernos de acuerdo en esto, así que por favor sé lo más claro y explícito posible. Trata de convencernos.

3. Como ya sabéis, a Construcciones Imposibles S. A. lo que más le importa son los costes económicos, ¿cuánto puede costar un accidente mortal a la empresa? Justifica tu respuesta.

4. Como es la actividad más arriesgada, interesa especialmente que propongas los tres equipos de protección individual que deberían usar los jardineros. Explica por qué eliges esos.

\begin{tabular}{|l|l|}
\hline Equipo de protección & Uso \\
\hline & \\
& - \\
\hline
\end{tabular}

5. Para que una empresa reduzca el número de accidentes laborales, ¿qué consejos deberíamos darles a los trabajadores?, ¿qué argumentos para que 
entiendan la importancia de cada consejo le darías a los trabajadores si fueses un técnico en prevención de riesgos laborales?

6. Selecciona los tres trabajos que consideras que son los más peligrosos en la construcción del puente. Identifica el tipo de riesgo al que crees que se ven expuestos los trabajadores en cada uno de ellos.

7. Hemos identificado una tasa alta de accidentes en este mes de agosto, ¿a qué se puede deber? Por favor, argumenta cada una de las razones

\section{Aplicación del CIMA}

En el ciclo de mejora se trataron cinco temas contemplados en el programa de la asignatura de IPRL, todos ellos dentro del contexto de la Seguridad en el Trabajo. Las sesiones se dividieron en siete, seis de ellas de una hora de duración (la sesión 1, 2, 3, 4, 6 y 7) y una sesión de dos horas (sesión 5), como se pudo observar en el apartado anterior.

La primera sesión se desarrolló en torno al concepto de accidente de trabajo. Esta sesión resultó muy productiva para los alumnos; a la sesión asistieron alrededor de 90 de ellos. El tiempo destinado a las explicaciones de la profesora y a la formulación de la pregunta fue suficiente, pero la formación de grupos de trabajo no fue sencilla. La plataforma limitaba a 20 grupos de trabajo; para el alto número de estudiantes resultó insuficiente para hacer los grupos que ellos mismo decidieron. Toda esta situación generó algo de confusión y provocó que se perdiera algo de tiempo en la realización de la actividad. Superado este pequeño obstáculo de forma, se trabajó por lo general muy bien. Los alumnos respondieron sin problemas a las preguntas y estuvieron en todo momento muy motivados.

La segunda sesión duró al igual que la primera una hora. Al contrario que en la primera, los estudiantes no

Ciclos de Mejora en el Aula (2020). Experiencias de Innovación Docente de la US Esta obra se distribuye con la licencia Creative Commons 
participaron activamente. Esta sesión se planteó como una sesión con carácter teórico, centrada en los conocimientos básicos sobre técnicas de seguridad. A pesar de que se pidió participación, los alumnos no respondían de forma fluida y no se plantearon dudas sobre los contenidos expuestos a los largo de la sesión 2. El tiempo dedicado a la sesión estuvo bien dimensionado.

En la tercera sesión, se trabajó por grupos una actividad de carácter práctico. Los alumnos aprendieron a calcular índices estadísticos en materia de seguridad laboral. Tras la breve explicación de las fórmulas que se emplearías en la sesión 3, los alumnos en grupos resolverían el problema propuesto. Las limitaciones de trabajo grupal mediante la plataforma limitó el buen funcionamiento de la actividad, ya que sólo se pudieron formar 20 grupos de alumnos. Muchos alumnos decidieron trabajar mediante Skype u otras herramientas para comunicarse entre ellos. En el desarrollo de esta actividad los alumnos no pararon de preguntar entre sí o de preguntar a la profesora. Al final no todos los alumnos pudieron terminar a tiempo los índices propuestos. El tiempo quizás resultó escaso.

Los fundamentos teóricos sobre investigación de accidentes de trabajo y sus costes se explicaron en la cuarta sesión. Los alumnos participaron ante las preguntas que se les iban formulando durante la explicación teórica del tema. En esta sesión con carácter más teórico participaron más estudiantes que en la sesión 2, que también era de contenido teórico. Esto hizo que el tiempo fuera insuficiente, ya que a cada alumno que quiso intervenir durante la sesión 4, se le cedió la palabra. Los alumnos por lo general se interesaron por el contenido de la sesión sobre los costes de la no prevención.

La quinta sesión fue la más larga, entorno a la técnica de seguridad de Investigación de Accidentes. Esta sesión se dividió en varias partes. La primera de ella, tras la breve explicación sobre la actividad a desarrollar, fue de formación de los grupos. Esta vez los alumnos, previo aviso de la profesora, ya tenían los grupos hechos y habían 
establecido canales para comunicarse sin limitaciones. En la segunda parte de esta sesión 5, los alumnos trabajaron muy bien y motivados, ya que los resultados de esta actividad iban a ser valorados significativamente en su evaluación final. La última parte se dedicó a la exposición de los trabajos y dudas. Por falta de tiempo sólo pudieron exponer muy pocos alumnos. Los alumnos que no expusieron se grabaron en video para que se les valorara la actividad.

En la sexta y séptima sesión no asistieron muchos estudiantes en comparación con la media de alumnos que solían asistir en sesiones anteriores (en torno a 90 alumnos). Sólo 60 estudiantes frente a las 111 matriculados. En la sesión 6, la descripción y explicación de conceptos básicos para realizar investigaciones de accidentes laborales se alargó algo más de lo planeado; sin embargo, el hecho de asistir menor número de alumnos, hizo más breve y sencilla la formación de los grupos de trabajo, por lo que el tiempo de la sesión al final estuvo bien dimensionado. Al concluir la sesión 6, un par de grupos expusieron los resultados de su evaluación de riesgos, de forma muy acertada.

Concluyendo la sesión 7, el número de alumnos descendió significativamente. El hecho de que los alumnos tuviesen exámenes de diciembre de otras asignaturas ese día contribuyó a ello. Los estudiantes que sí estuvieron presentes en la sesión, participaron de forma puntual, pero motivados por la profesora. El hecho de plantearles una actividad concreta hizo que estuvieran un poco desmotivados. Se les pidió y dio tiempo a los alumnos para realizar el cuestionario inicial una vez finalizado el CIMA, y entregarlo de forma anónima.

De los alumnos que asistieron a esta última sesión surgieron dudas en torno a los métodos de calificación de la asignatura. Un alumno que no pudo asistir a esta séptima sesión escribió un email valorando positivamente las actividades realizadas.

Ciclos de Mejora en el Aula (2020). Experiencias de Innovación Docente de la US Esta obra se distribuye con la licencia Creative Commons 
Para hacerse una idea de los resultados del ciclo de mejora se realizaron unas escaleras de aprendizaje siguiendo directrices marcadas en la bibliografía (Porlán 2017) algunas de las escaleras de aprendizaje se pueden ver en la Figura 4, Figura 5 y Figura 6. Estas escaleras se construyeron a partir de las respuestas de los cuestionarios realizados por los alumnos antes y después del ciclo de mejora. Por similitud entre las respuestas obtenidas, se realizaron agrupaciones de cuestionarios; estas agrupaciones representaron unos porcentajes determinados sobre el total de cuestionarios analizados.

1

\section{PREGUNTA 1}

¿Cómo podemos evitar al máximo posible los tipos de accidente de trabajo que se pueden dar en la construcción del puente? Explica con detalle tus propuestas

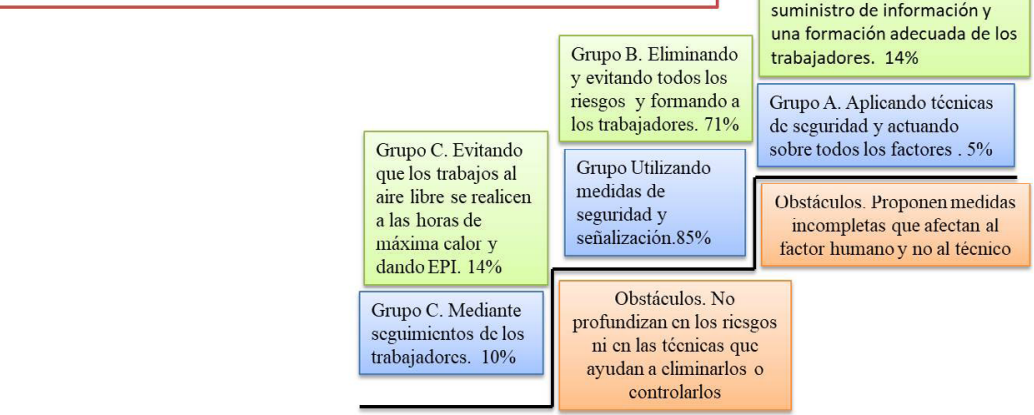

ANÁLISIS TRAS LAAPLICACIÓN DEL CIMA 2

Los alumnos han sabido discernir sobre la problemática planteada. La forma de expresarse en las

respuestas ha mejorado muchísimo. Han conseguido valorar los factores a tener en cuenta en la mayoría

de los cuestionarios. Es de destacar que en la $2^{\circ}$ ronda, el número de cuestionarios evaluado es mucho

menor, por eso los porcentajes no son del todo fiables

Figura 4. Escalera de aprendizaje para la pregunta 1

Los peldaños de las escaleras representas la diversidad de respuestas obtenidas, siendo las más próximas a un pensamiento abstracto aquellas dispuestas en los escalones más elevados. Por lo contrario, se agruparon y analizaron en el escalón inferior, aquellas respuestas que aportaban un pensamiento más concreto y menos profundo a la pregunta realizada. Como ejemplos más representativos, se muestran las siguientes escaleras.

Ciclos de Mejora en el Aula (2020). Experiencias de Innovación Docente de la US Esta obra se distribuye con la licencia Creative Commons 
En el caso de la pregunta 1 ¿Cómo podemos evitar al máximo posible los tipos de accidente de trabajo que se pueden dar en la construcción del puente?, en el cuestionario inicial antes del CIMA, muy pocos alumnos (5\%) fueron capaces de elaborar una respuesta con un pensamiento abstracto de la realidad, globalizando todos los factores de riesgo técnico y humano. En su mayoría (85\%) plantearon respuestas que incluían medidas concretas para trabajar sobre los factores de riesgo, siendo excesivamente teórica esta respuesta. Tras la realización del ciclo de mejora, este alto número de alumnos descendió hasta el 71\%, de ahí un grupo de alumnos superó el obstáculo que suponía la no percepción de la importancia del factor humano en los accidentes de trabajo. Con ello el número de alumnos en el último escaló llegó al 14\%. Hay que matizar, que los alumnos que no visualizaron la problemática de la pregunta, no cambiaron su respuesta. Esto puede observarse en la Figura 4.

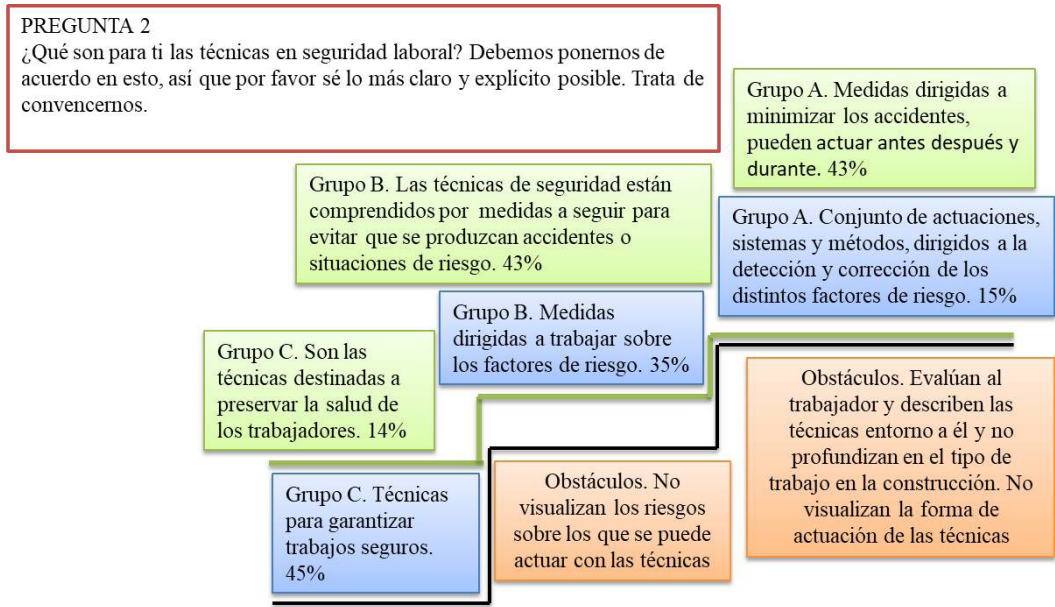

ANÁLISIS TRAS LA APLICACIÓN DEL CIMA 2

Todos los alumnos tras el CIMA 2 han mejorado considerablemente en sus respuestas. Tras la aplicación del CIMA ha asimilado el concepto de "técnicas de seguridad" y ha valorado no sólo el concepto sino la importancia que tienen las técnicas de seguridad en el ámbito de la prevención de riesgos laborales

Figura 5. Escalera de aprendizaje para la pregunta 2

El resultado del ciclo de mejora más significativo se puede ver en torno a la pregunta central ¿Qué son para

Ciclos de Mejora en el Aula (2020). Experiencias de Innovación Docente de la US Esta obra se distribuye con la licencia Creative Commons 
ti las técnicas en seguridad laboral?; esta cuestión era el centro del mapa de contenidos. Los alumnos después del ciclo de mejora mostraron sus conocimientos entorno a las técnicas de seguridad. Un porcentaje muy alto de alumnos (43\%) expusieron su juicio de forma global y abstracta, frente a la cuestión. Con el ciclo de mejora estos alumnos consiguieron alcanzar en nivel de conocimiento que les permitió responder superando el obstáculo que los bloqueaba en un nivel intermedio. Al observar como actúan las técnicas de seguridad frente a los accidentes de trabajo y al aprender la gran diversidad de técnicas que existen, sus respuestas fueron completas frente a las respuestas que aportaron antes del ciclo. En la Figura 5 pueden apreciarse los resultados obtenidos del análisis de esta pregunta 2.

Se podría decir que el mayor éxito del ciclo de mejora se pudo observar analizando la pregunta 5 Para que una empresa reduzca el número de accidentes laborales,... Todos los alumnos superaron el obstáculo relacionado con la importancia que tiene el factor humano en torno a un accidente de trabajo. Antes del ciclo de mejora, una cuarta parte de los alumnos no se planteaba la importancia de trabajar sobre el factor humano para evitar los accidentes laborales; sin embargo, gracias al trabajo realizado durante las sesiones de clase, este hecho quedo asumido por todos. Es importante observar como casi tres cuartas partes de los alumnos (71\%), tras la realización del ciclo, concluyeron en esta pregunta que la seguridad es responsabilidad de todos. Esta expresión está muy ligada a los contenidos actitudinales que se plantearon en el mapa de contenidos.

Como conclusión, las actividades que se realizaron, según algunos alumnos, ayudaron a asimilar de una forma más sencilla los conceptos sobre seguridad en el trabajo.

Ciclos de Mejora en el Aula (2020). Experiencias de Innovación Docente de la US Esta obra se distribuye con la licencia Creative Commons 


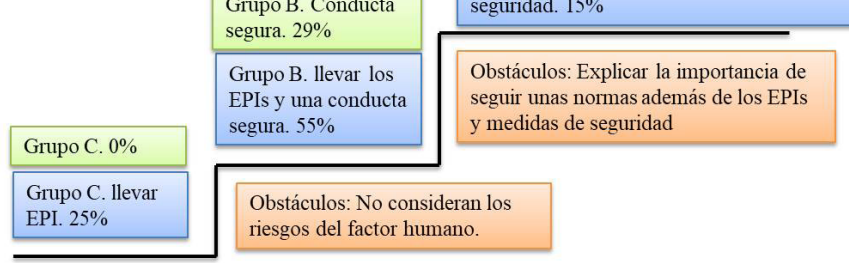

ANÁLISIS TRAS LA APLICACIÓN DEL CIMA 2

Todos los alumnos han superado el primer obstáculo tras la realización del CIMA. Han comprendido la gran

importancia que tiene el factor humano.

Figura 6. Escalera de aprendizaje para la pregunta 5

\section{Evaluación del CIMA puesto en práctica:}

Realizando una autoevaluación-evaluación del ciclo de mejora, considerando el aspecto relacionado con el aprendizaje de los estudiantes, no pensé que fuera importante el hecho de favorecer y generara un contexto de aprendizaje. El poder realizar con los alumnos trabajos grupales, que ellos compartan sus opiniones y sus ideas entorno a un tema, ha resultado muy motivador para ellos. En mi opinión, este ha sido el fuerte de mi CIMA, y estoy totalmente de acuerdo con lo que expone (Bruner, 1984) en el Aprendizaje por descubrimiento, en mi caso como profesora debo motivar a los estudiantes a que ellos mismos descubran relaciones entre conceptos y construyan proposiciones. En cuanto a los contenidos de enseñanza, fueron elegidos por su interconexión; el mapa de contenidos facilitó muchísimo la impartición de los mismos. He aprendido realizando el CIMA lo importante que es la diferenciación de los contenidos; además he comprendido que tiene valor el hecho de que los estudiantes asimilen no sólo los conceptos, sino también que valoren los contenidos procedimentales y actitudinales. Estos contenidos para mi estaban ocultos en la asignatura.

Ciclos de Mejora en el Aula (2020). Experiencias de Innovación Docente de la US Esta obra se distribuye con la licencia Creative Commons 
Reflexionando sobre la metodología escogida para la realización del ciclo de mejora, casi en su totalidad antes de la realización del CIMA se basaba en un modelo tradicional, compuesto por magistrales combinadas con resolución de problemas. El hecho de haber propuesto un modelo metodológico nuevo, cambiando las sesiones magistrales por actividades muy motivadoras para los estudiantes, ayudaron a generan un excelente contexto de aprendizaje para ellos. Partiendo de una actividad basada en una Pregunta Clave (PC), las sesiones se han desarrollado en un contexto participativo por parte de los alumnos, mediante Actividades Contrastadas (Ac) e Ideas de los Alumnos (IA). Gracias a estas actividades se han llegado a unas conclusiones del CIMA, que han permitido evaluar el grado de aprendizaje de los alumnos. Como autora del CIMA estoy muy satisfecha del diseño que al final apliqué, y que me ha reportado un resultado satisfactorio, viendo a los alumnos participando de forma fluida y constante en las sesiones.

Los principales obstáculos de los alumnos es la falta de cambio de visión de lo concreto a lo abstracto (Piaget, 1926). Los alumnos han sido capaces de llegar a niveles medios en las escaleras, superando obstáculos significativos (Vigotsky, 1978). Una vez realizado el ciclo de mejora, se ha notado levemente como cierto número de alumnos han mejorado sus respuestas acercándose más al pensamiento abstracto.

Como Modelo Didáctico Personal de referencia, me quedo con:

- Empleo de trabajos grupales en torno a un problema, donde la profesora ayuda a la superación de los obstáculos.

- Trabajos en grupos reducidos, donde la profesora no intervenga, siendo los propios alumnos los que trabajen sobre temas concretos

- Menor realización de clases magistrales

Ciclos de Mejora en el Aula (2020). Experiencias de Innovación Docente de la US Esta obra se distribuye con la licencia Creative Commons 
Palabras clave: Ingeniería de la Prevención de Riesgos Laborales, Ingeniería Mecánica, Docencia Universitaria y Experimentación Docente Universitaria

Keywords: Prevention Engineering of Occupational Risk, Mechanical Engineer, University Teaching and Experimentation in University Teaching

\section{Referencias bibliográficas}

Bain, K. (2004). What the Best College Teachers Do. Cambidge, MA: Hardvard University Press. (Trad. cast.: Lo que hacen los mejores profesores universitarios. Valencia: Publicaciones de la Universidad de Valencia).

Bruner, J. (1984). Acción, pensamiento y lenguaje. Madrid: Editores Alianza.

Finkel, D. (2000). Teaching with Your Moouth Shut. Portsmouth, NH: Heinemann Boynton/Cook. (Trad. cast.: Dar clase con la boca cerrada. Valencia: Publicaciones de la Universidad de Valencia, 2008).

Piaget, J. (1926). The language and thought of the child. New York: Harcourt, Brace, Jovanovich.

Porlán, R. (2017). Enseñanza Universitaria cómo mejorarla. Madrid: Ediciones Morata.

Vigotsky, L. (1978). Pensamiento y lenguaje. Madrid: Paidós.

Ciclos de Mejora en el Aula (2020). Experiencias de Innovación Docente de la US Esta obra se distribuye con la licencia Creative Commons 